\title{
The Asymptotic Solution of the Initial Boundary Value Problem to a Generalized Boussinesq Equation
}

\author{
Zheng Yin and Feng Zhang \\ Department of Applied Mathematics, Southwestern University of Finance and Economics, \\ Chengdu 610074, China
}

Correspondence should be addressed to Zheng Yin, yinzheng@swufe.edu.cn

Received 15 April 2012; Accepted 3 May 2012

Academic Editor: Shaoyong Lai

Copyright (c) 2012 Z. Yin and F. Zhang. This is an open access article distributed under the Creative Commons Attribution License, which permits unrestricted use, distribution, and reproduction in any medium, provided the original work is properly cited.

The $L^{2}$ space solution of an initial boundary problem for a generalized damped Boussinesq equation is constructed. Certain assumptions on the coefficients of the equation are found to show the existence and uniqueness of the global solution to the initial boundary problem. The explicit expression for the large time asymptotic solution is obtained.

\section{Introduction}

The classical Boussinesq equation can be expressed by

$$
u_{t t}=-\alpha u_{x x x x}+u_{x x}+\beta\left(u^{2}\right)_{x x^{\prime}}
$$

where $u(x, t)$ is the elevation of the free surface of fluid, the subscripts denote partial derivatives, and the constant coefficients $\alpha$ and $\beta$ depend on the depth of fluid and the characteristic speed of the long waves. The Boussinesq equation (1.1) was first derived by Boussinesq [1], in 1872, to describe propagation of long waves with small amplitude on the surface of shallow water. Since then, extensive research has been carried out to study the property and solutions of the equation and its associated initial boundary value problems. Clarkson [2] proposed a general approach for constructing exact solutions of (1.1). Hirota [3] deduced conservation laws and then examined the numerical solution of a Boussinesq equation. Yajima [4] investigated the nonlinear evolution of a linearly stable solution for (1.1). The exponentially decaying solution of the spherical Boussinesq equation has been 
investigated by Nakamura [5]. Galkin et al. [6] developed rational solutions of the onedimensional Boussinesq equation with both zero and nonzero boundary conditions at the infinity in the space from the known solutions of the Kadomtzev-Petviashvili equation. The structure of their solutions generalizes a family of rational solutions of the Korteweq-de Vries equation to the case of two-wave processes. For many other methods to investigate the Boussinesq system and other shallow water models, the reader is referred to [7-13] and the references therein.

Various generalizations of the classical Boussinesq equation have been proposed and studied from many aspects, in particular the well-posedness of the Cauchy problem for the following equation,

$$
u_{t t}=-u_{x x x x}+u_{x x}+(f(u))_{x x} .
$$

Bona and Sachs [14] showed that the special solitary wave solutions of (1.2) are nonlinearly stable for a range of wave speeds. The local and global well-posedness of the problem has been proved by transforming (1.2) into a system of nonlinear Schrödinger equations [15].

Varlamov [16] considered the initial-boundary condition for the following damped Boussinesq equation:

$$
u_{t t}-2 b u_{t x x}=-\alpha u_{x x x x}+u_{x x}+\beta\left(u^{2}\right)_{x x^{\prime}}
$$

where the second term on the left-hand side is responsible for dissipation, $\beta \in R^{1}$, and $\alpha$ and $b$ are assumed to be positive constants and satisfy the assumption that $\alpha>b^{2}$. An initialboundary value problem for (1.3) with small initial data is considered for the case of one space dimension. The classical solution of the problem is constructed, and the long-time asymptotics is obtained in explicit form. The asymptotics show the presence of both time and space oscillations and the exponential decay of the solution in time due to dissipations. Varlamov [17] has also used the eigenfunction expansion method to consider the long-time asymptotics for a damped Boussinesq equation in a ball which is similar to (1.3).

The aim of this paper is to study the initial-boundary value problem for the following generalized damped Boussinesq equation:

$$
u_{t t}-a u_{t t x x}-2 b u_{t x x}+\frac{2 b}{a} u_{t}=-c u_{x x x x}+u_{x x}-p^{2} u+\beta\left(u^{2}\right)_{x x^{\prime}}
$$

where $a, b$ and $c$ are positive constants and constants $p \geq 0$ and $\beta \in R^{1}$. We assume the cases $a+c>b^{2}$ and $p \geq \sqrt{2} b / a$. The classical solutions of the initial-boundary value problem for (1.4) will be constructed in the form of a Fourier series with coefficients in their own term represented as series in terms of a small parameter involved in the initial conditions. It is shown that the new solution of the initial-boundary value problem for (1.4) is wellposedness. In addition, the long time behavior of our solution also shows the presence of damped oscillations decaying exponentially in time as $t \rightarrow \infty$. 


\section{Theorem of Existence, Uniqueness, and Asymptotics}

In this paper, we consider the following initial-boundary value problem for the damped Boussinesq equation:

$$
\begin{gathered}
u_{t t}-a u_{t t x x}-2 b u_{t x x}+\frac{2 b}{a} u_{t}=-c u_{x x x x}+u_{x x}-p^{2} u+\beta\left(u^{2}\right)_{x x^{\prime}} \\
u(0, t)=u(\pi, t)=0, \quad t>0, \\
u_{x x}(0, t)=u_{x x}(\pi, t)=0, \quad t>0, \\
u(x, 0)=\varepsilon^{2} \varphi(x), \quad u_{t}(x, 0)=\varepsilon^{2} \psi(x), \quad x \in(0, \pi),
\end{gathered}
$$

where $a, b, c$, and $p$ are positive constants, $\beta \in R$, and $\varepsilon$ is a small parameter.

Definition 2.1. The function $u(x) \in C^{2 n}(0, \pi), n \geq 1$, if $u(0)=u(\pi)=u^{\prime \prime}(0)=u^{\prime \prime}(\pi)=\cdots=$ $u^{(2 n-2)}(0)=u^{(2 n-2)}(\pi)=0$ and $u^{(2 n)}(x) \in L^{2}(0, \pi)$.

Definition 2.2. The function $u(x, t)$ defined on $[0, \pi] \times[0,+\infty)$ is said to be the classical solution of the problem defined by (2.1), if it has two continuous derivatives on $[0, \pi] \times[0,+\infty)$ and satisfies system (2.1).

The main results obtained are summarized in the following theorem.

Theorem 2.3. If $a>0, a+c>b^{2}, p \geq \sqrt{2} b / a, \varphi(x) \in C^{8}(0, \pi), \psi(x) \in C^{6}(0, \pi)$, then there is $a$ $\varepsilon_{0}>0$ such that, for $0<\varepsilon \leq \varepsilon_{0}$, problem (2.1) has a unique classical solution of which is represented in the form of

$$
u(x, t)=\sum_{N=0}^{\infty} \varepsilon^{N+1} u^{(N)}(x, t),
$$

where the function $u^{(N)}(x, t)$ is as that defined by expressions (3.24) in the proof below. This series and the series for the derivatives of $u(x, t)$ involved in (2.1) converge absolutely and uniformly with respect to $x \in[0, \pi], t \geq 0, \varepsilon \in\left[0, \varepsilon_{0}\right]$. In addition, the solution of system (2.1) has the following long time behavior as $t \rightarrow+\infty$ :

$$
u(x, t)=e^{-(b / a) t}\left[(A \cos \sigma t+B \sin \sigma t) \sin x+O\left(e^{[(-\eta b / a) t]}\right)\right]
$$

where $\sigma=\sqrt{a c+\left(a+c-b^{2}\right)+\left(1+a p^{2}-\left(b^{2} / a^{2}\right)\right)+p^{2}-\left(b^{2} / a^{2}\right) / 1+a}$ is a positive constant, $0<\eta<1$, and the coefficients $A$ and $B$ are defined by (3.44).

\section{Proof of Theorem 2.3}

The proof of Theorem 2.3 includes three parts, namely, existence of a solution, uniqueness of the solution, and the asymptotics of the solution. The main techniques used in this paper are based on those presented in [16]. However, it should be emphasized that the method for proving uniqueness of the solution is different from that of [16]. 


\subsection{Existence of a Solution}

We make an odd extension of $u(x, t)$ in $x$ to the interval $[-\pi, 0]$ and represent $u$ in the form of a complex Fourier series; namely,

$$
u(x, t)=\sum_{\substack{n=-\infty \\ n \neq 0}}^{\infty} \widehat{u}_{n}(t) e^{i n x}, \quad \widehat{u}_{n}(t)=\frac{1}{2 \pi} \int_{-\pi}^{\pi} u(x, t) e^{-i n x} d x
$$

where $\widehat{u}_{-n}(t)=-\widehat{u}_{n}(t)$ for $n \geq 1$. For the above equalities, we shall use the fact that $u(x, t)$ belongs to the space $L^{2}(-\pi, \pi)$ for each fixed $t>0$, and we shall denote the corresponding norm by

$$
\|u(t)\|=\|u(t)\|_{L^{2}(-\pi, \pi)}=\left(\int_{-\pi}^{\pi}|u(x, t)|^{2} d x\right)^{1 / 2} .
$$

From (3.1), we obtain, through a simple calculation, that

$$
u(x, t)=2 i \sum_{n=1}^{\infty} \widehat{u}_{n}(t) \sin n x, \quad \widehat{u}_{n}(t)=\frac{1}{i \pi} \int_{0}^{\pi} u(x, t) \sin n x d x
$$

Noting the initial functions, we can write on $[-\pi, \pi]$ with

$$
\widehat{\varphi}_{-n}=\widehat{\varphi}_{n}, \quad \widehat{\psi}_{-n}=\widehat{\psi}_{n},
$$

for $n \geq 1$ that

$$
\varphi(x)=\sum_{\substack{n=-\infty \\ n \neq 0}}^{\infty} \widehat{\varphi}_{n} e^{i n x}, \quad \psi(x)=\sum_{\substack{n=-\infty \\ n \neq 0}}^{\infty} \widehat{\psi}_{n} e^{i n x}
$$

Then on the interval $[0, \pi]$, we obtain

$$
\begin{aligned}
\varphi(x) & =2 i \sum_{n=1}^{\infty} \widehat{\varphi}_{n} \sin n x, \quad \psi(x)=2 i \sum_{n=1}^{\infty} \widehat{\psi}_{n} \sin n x, \\
\widehat{\varphi}_{n} & =\frac{1}{i \pi} \int_{0}^{\pi} \varphi(x) \sin n x d x, \quad \widehat{\psi}_{n}=\frac{1}{i \pi} \int_{0}^{\pi} \psi(x) \sin n x d x .
\end{aligned}
$$


Integrating (3.6) by parts and using the smoothness assumption of the initial data, we get the following inequalities:

$$
\left|\widehat{\varphi}_{n}\right| \leq C_{1} n^{-8}, \quad\left|\widehat{\psi}_{n}\right| \leq C_{1} n^{-6}, \quad n \geq 1,
$$

where $C_{1}$ is a positive constant. Substituting (3.1) and (3.5) into (2.1), we have the following Cauchy problem for the function $\widehat{u}_{n}(t), n \in Z$ :

$$
\begin{gathered}
\left(1+a n^{2}\right) \widehat{u}_{n}^{\prime \prime}(t)+2\left(b n^{2}+\frac{b}{a}\right) \widehat{u}_{n}^{\prime}(t)+\left(c n^{4}+n^{2}+p^{2}\right) \widehat{u}_{n}(t)=-\beta n^{2} P\left(\widehat{u}_{n}(t)\right), \\
\widehat{u}_{n}(0)=\varepsilon^{2} \widehat{\varphi}_{n}, \quad \widehat{u}_{n}^{\prime}(0)=\varepsilon^{2} \widehat{\psi}_{n}
\end{gathered}
$$

where

$$
P\left(\widehat{u}_{n}(t)\right)=\sum_{\substack{g=-\infty \\ g \neq 0, n}}^{\infty} \widehat{u}_{n-g}(t) \widehat{u}_{g}(t), \quad \widehat{u}_{-n}(t)=-\widehat{u}_{n}(t) \quad \text { for } n \geq 1 .
$$

For $n=1$, we have

$$
P\left(\widehat{u}_{1}(t)\right)=2 \sum_{g=1}^{\infty} \widehat{u}_{-g}(t) \widehat{u}_{1+g}(t)=-2 \sum_{g=1}^{\infty} \widehat{u}_{g}(t) \widehat{u}_{1+g}(t) .
$$

For $n \geq 2$, it has

$$
P\left(\widehat{u}_{n}(t)\right)=\sum_{g=1}^{n-1} \widehat{u}_{n-g}(t) \widehat{u}_{g}(t)-2 \sum_{g=1}^{\infty} \widehat{u}_{g}(t) \widehat{u}_{n+g}(t)
$$

Letting $\Phi_{n}=\varepsilon \widehat{\varphi}_{n}, \Psi_{n}=\varepsilon \widehat{\psi}_{n}$ and solving the initial value problem (3.8), we get

$$
\begin{aligned}
\widehat{u}_{n}(t)= & \varepsilon e^{-(b / a) t}\left\{\left[\cos \left(\sigma_{n} t\right)+\frac{b}{a} \cdot \frac{\sin \left(\sigma_{n} t\right)}{\sigma_{n}}\right] \Phi_{n}+\frac{\sin \left(\sigma_{n} t\right)}{\sigma_{n}} \Psi_{n}\right\} \\
& -\frac{\beta n^{2}}{\sigma_{n}\left(1+a n^{2}\right)} \int_{0}^{t} \exp \left[-\frac{b}{a}(t-\tau)\right] \sin \left[\sigma_{n}(t-\tau)\right] P\left(\widehat{u}_{n}(\tau)\right) d \tau,
\end{aligned}
$$

where

$$
\begin{array}{r}
\sigma_{n}=\frac{\sqrt{a c n^{6}+\left(a+c-b^{2}\right) n^{4}+\left(1+a p^{2}-(2 b / a)\right) n^{2}+p^{2}-\left(b^{2} / a^{2}\right)}}{1+a n^{2}} \\
n \geq 1, \quad a+c>b^{2}, \quad p \geq \frac{\sqrt{2} b}{a} .
\end{array}
$$


Now, in order to solve the integral equation, we use the perturbation theory. Firstly, we express $\widehat{u}_{n}(t), n \geq 1$, as a formal series in $\varepsilon$ :

$$
\widehat{u}_{n}(t)=\sum_{N=0}^{\infty} \varepsilon^{N+1} \widehat{\xi}_{n}^{(N)}(t)
$$

Then, by substituting (3.14) into (3.12) and equating the coefficients of the like powers of $\varepsilon$, we get the following formulas, respectively, for $N=0$ and $N \geq 1$ :

$$
\begin{gathered}
\widehat{\xi}_{n}^{(0)}(t)=\varepsilon e^{-(b / a) t}\left\{\left[\cos \left(\sigma_{n} t\right)+\frac{b}{a} \cdot \frac{\sin \left(\sigma_{n} t\right)}{\sigma_{n}}\right] \Phi_{n}+\frac{\sin \left(\sigma_{n} t\right)}{\sigma_{n}} \Psi_{n}\right\}, \\
\widehat{\xi}_{n}^{(N)}(t)=-\frac{\beta n^{2}}{\sigma_{n}\left(1+a n^{2}\right)} \int_{0}^{t} \exp \left[-\frac{b}{a}(t-\tau)\right] \times \sin \left[\sigma_{n}(t-\tau)\right] Q_{N}\left(\widehat{\xi}_{n}^{(j)}(\tau)\right) d \tau, \quad N \geq 1,
\end{gathered}
$$

where $n \geq 1$ and

$$
Q_{N}\left(\widehat{\xi}_{n}^{(j)}(\tau)\right)=\varepsilon_{n} \sum_{g=1}^{n-1} \sum_{j=1}^{N} \widehat{\xi}_{n-g}^{(j-1)}(\tau) \widehat{\xi}_{g}^{(N-j)}(\tau)-2 \sum_{g=1}^{\infty} \sum_{j=1}^{N} \widehat{\xi}_{n+g}^{(j-1)}(\tau) \widehat{\xi}_{g}^{(N-j)}(\tau)
$$

in which $\varepsilon_{1}=0$ and $\varepsilon_{n}=1$ for $n \geq 2$.

Now we must prove that the formally constructed function (3.3), together with (3.12)(3.14), does represent a solution of problem (2.1). To do this, we shall show that the series

$$
\begin{aligned}
u(x, t) & =\sum_{\substack{n=-\infty \\
n \neq 0}}^{\infty} e^{i n x} \sum_{N=0}^{\infty} \varepsilon^{N+1} \widehat{\xi}_{n}^{(N)}(t) \\
& =2 i \sum_{n=1}^{\infty} \sin n x \sum_{N=0}^{\infty} \varepsilon^{N+1} \widehat{\xi}_{n}^{(N)}(\tau)
\end{aligned}
$$

converges absolutely and uniformly. For this purpose, we firstly establish the following time estimates for $n \geq 1, t>0, N \geq 0$ :

$$
\left|\widehat{\xi}_{n}^{(N)}(t)\right| \leq C^{N}(N+1)^{-2} n^{-6} e^{-(b / a) t} .
$$

Here and in the sequel we denote by $C$ any positive constant independent of $N, n, \varepsilon$, and $t$, but possibly depending on the coefficients of the equation and the initial functions.

We shall use the induction on the number $N$. For $N=0$, we have from (3.7) and (3.15) that

$$
\begin{aligned}
\left|\widehat{\xi}_{n}^{(0)}(t)\right| & \leq \varepsilon e^{-(b / a) t}\left[\left(1+\frac{b}{a \sigma_{n}}\right)\left|\widehat{\Phi}_{n}\right|+\frac{1}{\sigma_{n}}\left|\Psi_{n}\right|\right] \\
& \leq \varepsilon n^{-6} e^{-(b / a) t} .
\end{aligned}
$$


Assuming that (3.19) is valid for all $\hat{\xi}_{n}^{(s)}(t)$ with $0 \leq s \leq N-1$, we shall prove that (3.19) also holds for $s=N$. According to [16], for any integer $n \geq 1, g \geq 1$, and $g \neq n$, we have

$$
\begin{gathered}
|n-g|^{-6} g^{-6} \leq 2^{6} n^{-6}\left[g^{-6}+|n-g|^{-6}\right], \\
j^{-2}(N+1-j)^{-2} \leq 2^{2}(N+1)^{-2}\left[j^{-2}+(N+1-j)^{-2}\right] .
\end{gathered}
$$

From (3.16) and noting that

$$
\frac{b n^{2}}{1+a n^{2}} \geq \frac{b}{a} \text { for } n \neq 0
$$

we have

$$
\begin{aligned}
\left|\widehat{\xi}_{n}^{(N)}(t)\right| \leq & C|\beta|(N+1)^{-2} n^{-6} \sum_{g=1}^{\infty}\left(g^{-6}+|n-g|^{-6}\right) \\
& \times \sum_{j=1}^{\infty} C^{j-1} C^{N-j}\left[(N+1-j)^{-2}+j^{-2}\right]\left|S_{N}(n, t)\right|,\left|S_{N}(n, t)\right| \\
\leq & C e^{-(b / a) t} \int_{0}^{t} e^{((b / a)-(2 b / a)) \tau} d \tau \\
\leq & C e^{-(b / a) t} \times\left|\frac{e^{((b / a)-(2 b / a) t}-1}{(b / a)-(2 b / a)}\right| \\
\leq & C e^{-(b / a) t} .
\end{aligned}
$$

Therefore, we know that inequality (3.19) holds.

For the derivation of (3.15), we recall (3.3) and (3.14) with $\widehat{\xi}_{n}^{(N)}$ defined by (3.15) and (3.16) and interchange the order of summations in the series; namely,

$$
u(x, t)=2 i \sum_{n=1}^{\infty} \widehat{u}_{n}(t) \sin n x=2 i \sum_{n=1}^{\infty} \sin n x \sum_{N=0}^{\infty} \varepsilon^{N+1} \widehat{\xi}_{n}^{(N)}(t)=\sum_{N=0}^{\infty} \varepsilon^{N+1} u^{(N)}(x, t),
$$

where

$$
u^{(N)}(x, t)=2 i \sum_{n=1}^{\infty} \widehat{\xi}_{n}^{(N)} \sin n x
$$


The interchange of the order of summations is allowable as the series is absolutely and uniformly convergent for $x \in[0, \pi], t \geq 0$ and $\varepsilon \in\left[0, \varepsilon_{0}\right]$. Differentiating (3.15) and (3.16), we get, for $k=1,2$, that

$$
\begin{gathered}
\partial_{k}^{t} \widehat{\xi}_{n}^{(0)}(t)=\sum_{l=0}^{k} c_{k}^{l}(-1)^{l}\left(\frac{b}{a}\right)^{l} e^{-(b / a) t} \partial_{k}^{k-l}\left\{\left[\cos \left(\sigma_{n} t\right)+\frac{b}{a} \frac{\sin \left(\sigma_{n} t\right)}{\sigma_{n}}\right] \Phi_{n}+\frac{\sin \left(\sigma_{n} t\right)}{\sigma_{n}} \Psi_{n}\right\}, \\
\partial_{t}^{k} \widehat{\xi}_{n}^{(N)}(t)=-\frac{\beta n^{2}}{\left(1+a n^{2}\right) \sigma_{n}} \int_{0}^{t} g_{k}(n, t-\tau) Q_{N}\left(\widehat{\xi}_{n}^{(j)}(\tau)\right) d \tau+R_{k}(n, t),
\end{gathered}
$$

where

$$
g_{k}(n, t)=\sum_{l=0}^{k} c_{k}^{l}(-1)^{l}\left(\frac{b}{a}\right)^{l} e^{-(b / a) t} \sigma_{n}^{k-l} \sin \left[\sigma_{n} t+\frac{(k-l) \pi}{2}\right]
$$

$Q_{N}\left(\widehat{\xi}_{n}^{(j)}(\tau)\right)$ is defined by $(3.16), c_{k}^{l}(l=0,1, \ldots, k)$ are binomial coefficients, and $R_{k}(n, t)(k=$ $1,2)$ are obtained by differentiating the integral in (3.16) and are as follows:

$$
R_{1}(n, t)=0, \quad R_{2}(n, t)=-\beta n^{2} Q_{N}\left(\widehat{\xi}_{n}^{(j)}(t)\right) .
$$

Hence, using (3.19), we deduce that for, $n \geq 1, N \geq 0, t>0$, and $k \geq 0,1,2$,

$$
\begin{gathered}
\left|\partial_{t}^{k} \widehat{\xi}_{n}^{(N)}(t)\right| \leq C^{N}(N+1)^{-2} n^{2 k-6} e^{-(b / a) t}, \\
\left|\partial_{t}^{k} \widehat{u}_{n}(t)\right| \leq C n^{2 k-6} e^{-(b / a) t} .
\end{gathered}
$$

Using these estimates and calculating the necessary derivatives of (3.29), we can prove straightforwardly that (3.29) represents the classical solution of the initial-boundary value problem defined by system (2.1).

\subsection{Uniqueness of the Solution}

For proving the uniqueness of the constructed solution, we shall assume that there exist two classical solutions $u^{(1)}(x, t)$ and $u^{(2)}(x, t)$ to system (2.1) and then deduce that $u^{(1)}(x, t)$ must be equal to $u^{(2)}(x, t)$. 
Making an odd extension of the two solutions to the segment $(-\pi, 0]$, we notice that both of them belong to the $L^{2}(-\pi, \pi)$ space, and according to Definition 2.2, we have for each fixed time $t>0$ that

$$
\max _{x \in[-\pi, \pi]}\left|u^{(1)}(x, t)\right| \leq C_{t}, \quad \max _{x \in[-\pi, \pi]}\left|u^{(2)}(x, t)\right|<C_{t}
$$

where $C_{t}$ is a constant depending on $t$. Let $w(x, t)=u^{(1)}(x, t)-u^{(2)}(x, t)$, and make an even extension of $w(x, t)$ to $\ldots(-3 \pi,-2 \pi),(-2 \pi,-\pi),(\pi, 2 \pi),(2 \pi, 3 \pi), \ldots$ Then, we have from (2.1) that

$$
\begin{gathered}
w_{t t}-a w_{t t x x}-2 b w_{t x x}+\frac{2 b}{a} w_{t}=-c w_{x x x x}+w_{x x}-p^{2} w+\beta\left[w(x, t)\left(u^{(1)}(x, t)+u^{(2)}(x, t)\right)\right]_{x x^{\prime}} \\
w(x, 0)=w_{t}(x, t)=0 .
\end{gathered}
$$

Taking the Fourier transform of $w$ in the interval $(-\infty,+\infty)$; namely,

$$
\widehat{w}(\xi, t)=\int_{-\infty}^{+\infty} e^{-i x \xi} w(x, t) d x
$$

we have

$$
\left(1+a \xi^{2}\right) \widehat{w}^{\prime \prime}(\xi, t)+2 b\left(\xi^{2}+\frac{b}{a}\right) \widehat{w}(\xi, t)+\left(c \xi^{4}+\xi^{2}+p^{2}\right) \widehat{w}(\xi, t)=-\beta \xi^{2} \widehat{f}(\xi, t),
$$

where

$$
f(x, t)=w\left(u^{(1)}+u^{(2)}\right)(x, t) .
$$

From (3.33), we have

$$
\widehat{w}(\xi, t)=-\frac{\beta \xi^{2}}{\sigma_{\xi}\left(1+a \xi^{2}\right)} \int_{0}^{t} \exp \left[-\frac{b}{a}(t-\tau)\right] \sin \left(\sigma_{\xi}(t-\tau)\right) \widehat{f}(\xi, \tau) d \tau,
$$

where

$$
\sigma_{\xi}=\frac{\sqrt{a c \xi^{6}+(a+c-b) \xi^{4}+\left(1+a p^{2}-\left(2 b^{2} / a\right)\right) \xi^{2}+p^{2}-\left(b^{2} / a^{2}\right)}}{1+a \xi^{2}} .
$$


Thus, it has

$$
\begin{aligned}
|\widehat{w}(\xi, t)| & \leq C \int_{0}^{t}\left|\exp \left[-\frac{b}{a}(t-\tau)\right] \widehat{f}(\xi, \tau)\right| d \tau \\
& \leq C\left[\int_{0}^{t} \exp \left[-\frac{b}{a}(t-\tau)\right] d \tau\right]^{1 / 2}\left[\int_{0}^{t}|\widehat{f}(\xi, \tau)|^{2} d \tau\right]^{1 / 2} \\
& \leq C\left[\int_{0}^{t}|\widehat{f}(\xi, \tau)|^{2} d \tau\right]^{1 / 2} .
\end{aligned}
$$

It follows from (3.37) and the Parseval inequality that

$$
\begin{aligned}
\int_{-\infty}^{+\infty}|\widehat{w}(\xi, t)|^{2} d \xi & \leq C \int_{-\infty}^{+\infty} \int_{0}^{t}|\widehat{f}(\xi, \tau)|^{2} d \tau d \xi \\
& \leq C \int_{0}^{t}\|\widehat{f}(\xi, t)\|_{L^{2}}^{2} d \tau \\
& \leq C \int_{0}^{t}\left\|w(x, \tau)\left(u^{(1)}(x, \tau)+u^{(2)}(x, \tau)\right)\right\|_{L^{2}}^{2} d \tau \\
& \leq C \int_{0}^{t} C_{\tau}\|w(x, \tau)\|_{L^{2}}^{2} d \tau .
\end{aligned}
$$

Using the Gronwall inequality, we obtain

$$
w(x, t)=0 \quad\left(\text { in } L^{2}\right)
$$

which implies that $u^{(1)}(x, t) \equiv u^{(2)}(x, t)$ and thus the solution is unique.

\subsection{Long Time Asymptotics}

To find the long-time behavior of the constructed solution, we firstly determine a subtle asymptotic estimate of $\widehat{u_{1}}(t)$ which will contribute to the major term and then estimate the remaining terms $\sum_{n=2}^{\infty} \widehat{u_{n}}(t) \sin n x$.

Since

$$
\widehat{u_{1}}(t)=\sum_{N=0}^{\infty} \varepsilon^{N+1} \widehat{\xi}_{1}^{(N)}(t)
$$


we substitute it into the integral equations (3.15) and (3.16) to obtain

$$
\begin{aligned}
& \hat{\xi}_{1}^{(0)}(t)=e^{-(b / a) t}\left[A^{(0)} \cos (\sigma t)+B^{(0)} \sin (\sigma t)\right], \\
& \hat{\xi}_{1}^{(N)}(t)=e^{-(b / a) t}\left\{\left[A^{(N)}+R_{A}^{(N)}(t)\right] \cos (\sigma t)+\left[B^{(N)}+R_{B}^{(N)}(t)\right] \sin (\sigma t)\right\},
\end{aligned}
$$

where

$$
\begin{aligned}
A^{(0)} & =\varepsilon \widehat{\varphi_{1}}, \\
B^{(0)} & =\frac{\varepsilon}{\sigma}\left(\frac{b}{1+a} \widehat{\varphi_{1}}+\widehat{\psi_{1}}\right), \\
\sigma & =\frac{\sqrt{a c+1+a+c-b^{2}}}{1+a}, \\
A^{(N)} & =\frac{\beta}{\sigma(1+a)} \int_{0}^{t} e^{(b / a) \tau} \sin (\sigma \tau) S_{u_{n}}(\tau) d \tau, \\
B^{(N)} & =-\frac{\beta}{\sigma(1+a)} \int_{0}^{t} e^{(b / a) \tau} \cos (\sigma \tau) S_{u_{n}}(\tau) d \tau, \\
R_{A}^{(N)} & =\frac{\beta}{\sigma(1+a)} \int_{0}^{+\infty} e^{(b / a) \tau} \sin (\sigma \tau) S_{u_{n}}(\tau) d \tau, \\
R_{B}^{(N)} & =-\frac{\beta}{\sigma(1+a)} \int_{0}^{+\infty} e^{(b / a) \tau} \cos (\sigma \tau) S_{u_{n}}(\tau) d \tau, \\
S_{u_{n}}(t) & =\sum_{g=1}^{\infty} \sum_{j=1}^{N} \widehat{\xi}_{1+g}^{(j-1)}(t) \widehat{\xi}_{g}^{(N-j)}(t), \quad N \geq 1,
\end{aligned}
$$

and the function $\widehat{\xi}^{(j)}(t), j=0,1, \ldots, N-1$ are defined by (3.15)-(3.16). Taking $n \geq 2, N \geq 1$ and using a method similar to that used in [16], it follows from (3.19) and (3.24) that there exists a positive number $0<\eta<1$ such that

$$
\left|R_{A}^{(N)}(t)\right| \leq c e^{-(\eta b t / a)}, \quad\left|R_{B}^{(N)}(t)\right| \leq c e^{-(\eta b t / a)}
$$

Hence, we have proved that, as $t \rightarrow+\infty$,

$$
\begin{gathered}
2 i \widehat{u_{1}}(t)=e^{-(b / a) t}[A \cos (\sigma t)+B \sin (\sigma t)]+O\left(e^{-((1+\eta) b / a) t}\right), \\
A=2 i \sum_{N=0}^{\infty} \varepsilon^{N+1} A^{(N)}, \quad B=2 i \sum_{N=0}^{\infty} \varepsilon^{N+1} B^{(N)},
\end{gathered}
$$

where $0<\eta<1, A^{(N)}$ and $B^{(N)}$ are defined by (3.41), and the series above converge absolutely and uniformly for $\varepsilon \in\left[0, \varepsilon_{0}\right]$. 
Now, we can represent the solution by

$$
\begin{aligned}
u(x, t) & =2 i \widehat{u_{1}}(t)+R_{u}(x, t), \\
R_{u}(x, t) & =2 i \sum_{n=2}^{\infty} \sin n x \sum_{N=0}^{\infty}{\widehat{\xi_{n}}}^{(N)}(t) .
\end{aligned}
$$

Using (3.24), we deduce that

$$
\begin{array}{r}
\left|R_{u}(x, t)\right| \leq \exp \left[\frac{-\eta b}{a} t\right] \sum_{n=0}^{\infty} c^{N} \varepsilon^{N+1}(N+1)^{-2} \sum_{N=2}^{\infty} n^{-6} \leq c \exp \left[\frac{-\eta b}{a} t\right], \\
u(x, t)=e^{-(b / a) t}\left[(A \cos \sigma t+B \sin \sigma t) \sin x+O\left(e^{-[(\eta b / a) t]}\right)\right], \quad 0<\eta<1 .
\end{array}
$$

It follows from (3.47) that the inequality (2.3) holds.

\section{Acknowledgments}

This work is supported by the Fundamental Research Funds for Central Universities (SWUFE 2012).

\section{References}

[1] J. Boussinesq, "Theorie des ondes et de remous qui se propadent le long d'un canal recangulaier horizontal, et communiquant au liquide contene dans ce cannal des vitesses sensiblement pareilles de la surface au fond," Journal de Mathématiques Pures et Appliquées, vol. 17, no. 2, pp. 55-108, 1872.

[2] P. A. Clarkson, "New exact solutions of the Boussinesq equation," European Journal of Applied Mathematics, vol. 1, no. 3, pp. 279-300, 1990.

[3] R. Hirota, "Solutions of the classical Boussinesq equation and the spherical Boussinesq equation: the Wronskian technique," Journal of the Physical Society of Japan, vol. 55, no. 7, pp. 2137-2150, 1986.

[4] N. Yajima, "On a growing mode of the Boussinesq equation," Progress of Theoretical Physics, vol. 69, no. 2, pp. 678-680, 1983.

[5] A. Nakamura, "Exact solitary wave solutions of the spherical Boussinesq equation," Journal of the Physical Society of Japan, vol. 54, no. 11, pp. 4111-4114, 1985.

[6] V. M. Galkin, D. E. Pelinovsky, and Y. A. Stepanyants, "The structure of the rational solutions to the Boussinesq equation," Physica D, vol. 80, no. 3, pp. 246-255, 1995.

[7] M. Tsutsumi and T. Matahashi, "On the Cauchy problem for the Boussinesq type equation," Mathematica Japonica, vol. 36, no. 2, pp. 371-379, 1991.

[8] V. Varlamov, "On the Cauchy problem for the damped Boussinesq equation," Differential and Integral Equations, vol. 9, no. 3, pp. 619-634, 1996.

[9] V. V. Varlamov, "On the damped Boussinesq equation in a circle," Nonlinear Analysis. Theory, Methods $\mathcal{E}$ Applications A, vol. 38, no. 4, pp. 447-470, 1999.

[10] H. A. Levine and B. D. Sleeman, "A note on the nonexistence of global solutions of initial-boundary value problems for the Boussinesq equation $u_{t t}=3 u_{x x x x}+u_{x x}-12\left(u^{2}\right)_{x x}$, Journal of Mathematical Analysis and Applications, vol. 107, no. 1, pp. 206-210, 1985.

[11] S. Y. Lai and Y. W. Wu, "Global solutions and blow-up phenomena to a shallow water equation," Journal of Differential Equations, vol. 249, no. 3, pp. 693-706, 2010.

[12] S. Y. Lai and Y. H. Wu, "A model containing both the Camassa-Holm and Degasperis-Procesi equations," Journal of Mathematical Analysis and Applications, vol. 374, no. 2, pp. 458-469, 2011. 
[13] T. J. Bridges and G. Derks, "Linear instability of solitary wave solutions of the Kawahara equation and its generalizations," SIAM Journal on Mathematical Analysis, vol. 33, no. 6, pp. 1356-1378, 2002.

[14] J. L. Bona and R. L. Sachs, "Global existence of smooth solutions and stability of solitary waves for a generalized Boussinesq equation," Communications in Mathematical Physics, vol. 118, no. 1, pp. 15-29, 1988.

[15] J. L. Bona and L. Luo, "More results on the decay of solutions to nonlinear, dispersive wave equations," Discrete and Continuous Dynamical Systems, vol. 1, no. 2, pp. 151-193, 1995.

[16] V. V. Varlamov, "On the initial-boundary value problem for the damped Boussinesq equation," Discrete and Continuous Dynamical Systems, vol. 4, no. 3, pp. 431-444, 1998.

[17] V. Varlamov, "Eigenfunction expansion method and the long-time asymptotics for the damped Boussinesq equation," Discrete and Continuous Dynamical Systems, vol. 7, no. 4, pp. 675-702, 2001. 


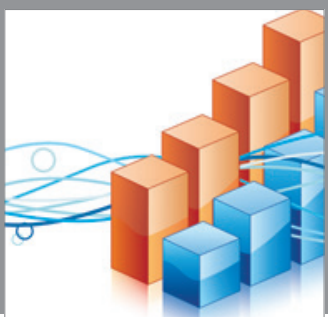

Advances in

Operations Research

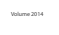

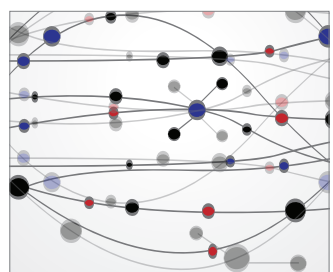

\section{The Scientific} World Journal
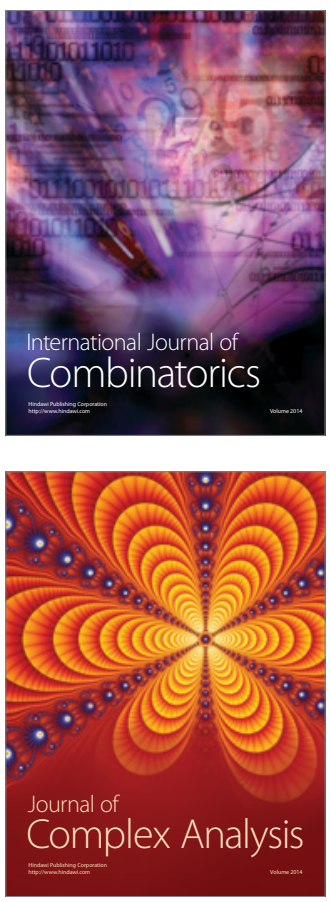

International Journal of

Mathematics and

Mathematical

Sciences
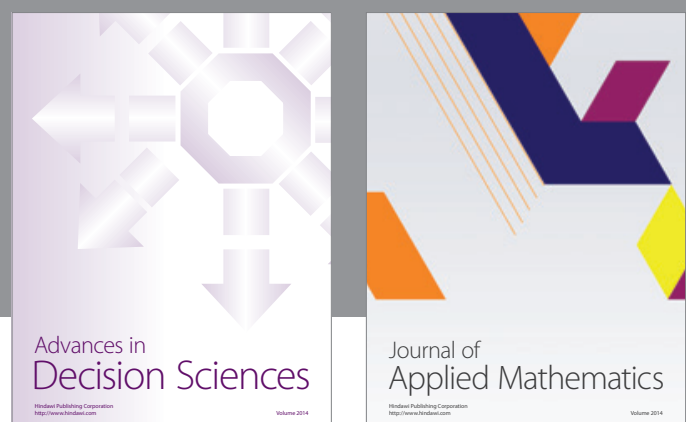

Journal of

Applied Mathematics
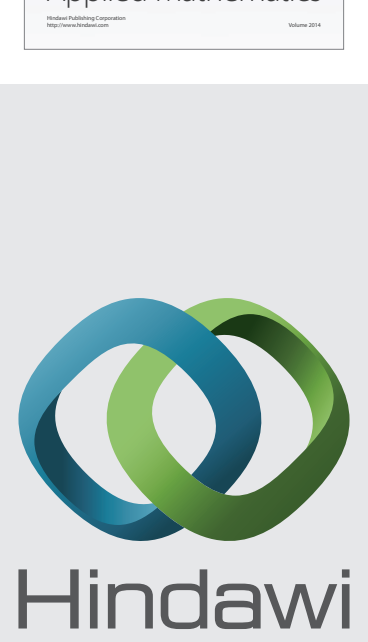

Submit your manuscripts at http://www.hindawi.com
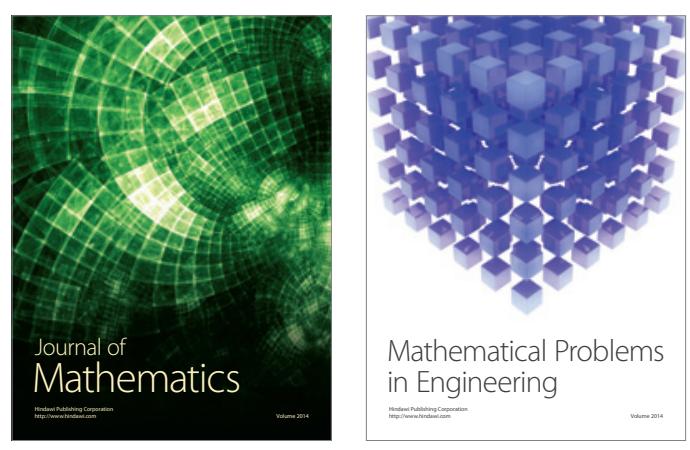

Mathematical Problems in Engineering
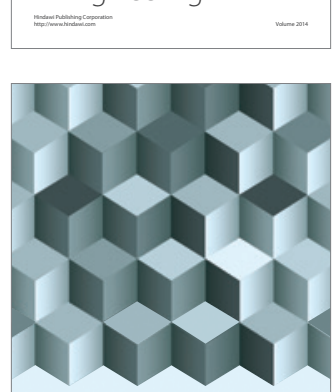

Journal of

Function Spaces
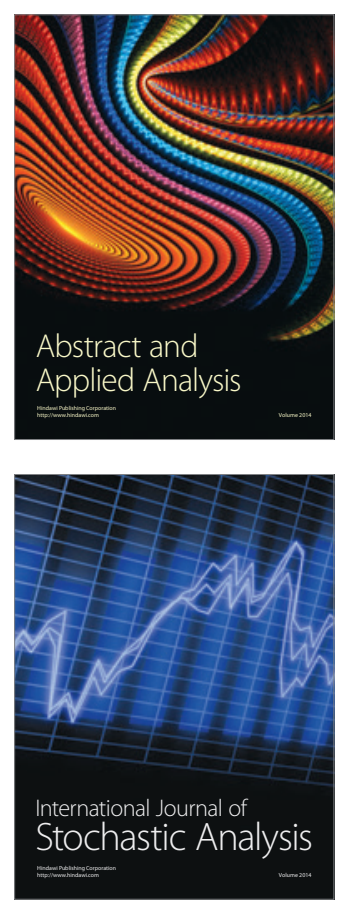

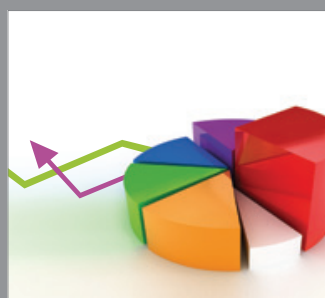

ournal of

Probability and Statistics

Promensencen
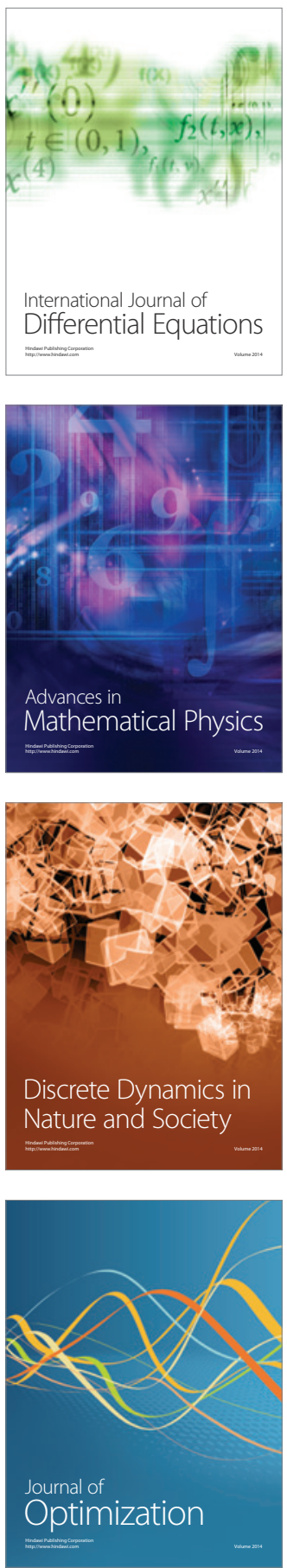